\title{
Unrecognised obstructive sleep apnoea is common in severe peripheral arterial
} disease

\author{
Karri T. Utriainen*, Juhani K. Airaksinen*, Olli Polo*, , Olli T. Raitakari', Mikko \\ J. Pietilä*, Harry Scheinin ${ }^{\S}$, Hans Y. Helenius ${ }^{f}$, Kari A. Leino ${ }^{\S}$, Erkki S. Kentala ${ }^{\S}$, \\ Jouko R. Jalonen ${ }^{\S}$, Harri Hakovirta**, Tiina M. Salo* and Timo T. Laitio ${ }^{\S}$
}

ABSTRACT: Patients needing surgery for peripheral arterial disease (PAD) represent a severe form of atherosclerosis with an overall 5 -yr mortality of $30 \%$ after revascularisation. The aetiology for poor post-operative clinical outcome in these high-risk patients is not fully established. Obstructive sleep apnoea (OSA) is associated with atherosclerosis and is an independent risk factor for fatal and nonfatal cardiac events. Here, we determine the prevalence of undiagnosed OSA in a homogenous group of PAD patients undergoing subinguinal surgical revascularisation.

82 consecutive patients (mean age $67 \pm 9 \mathrm{yrs}, 52$ males) with sinus rhythm and without congestive heart failure or previously diagnosed OSA were enrolled for pre-operative polysomnography and echocardiography.

OSA was present in $70(85 \%)$ patients (95\% Cl 75-93\%), of whom $24(34 \%)$ had severe OSA. OSA was mostly asymptomatic, and age- and sex-adjusted multivariate regression analysis showed no relation to obesity, metabolic syndrome or any manifestation of atherosclerosis, other than PAD. Left ventricular ejection fraction $(p=0.002)$ and high-density lipoprotein/total cholesterol ratio $(p=0.03)$ were the only independent predictors for the severity of OSA.

Thus, prevalence of OSA is unexpectedly high in patients with PAD and is not related to classical risk factors of sleep apnoea.

KEYWORDS: Cardiovascular disease, obstructive sleep apnoea

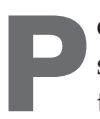
eripheral arterial disease (PAD) is an atherosclerotic syndrome indicating severe systemic vascular disease [1]. Patients needing surgery for PAD have a poor prognosis with an overall 5-yr mortality of $30 \%$ after revascularisation $[1,2]$. The aetiology and pathophysiological mechanisms for the poor post-operative clinical outcome in PAD patients is not well established.

Severe untreated obstructive sleep apnoea (OSA) is considered as an independent risk factor for cardiovascular mortality mainly due to myocardial infarction and stroke [3-6]. The prevalence of OSA is estimated to be nearly $30 \%$ in middle-aged unselected population, but it is much higher $(\sim 50 \%)$ in populations with cardiovascular disease. $>85 \%$ of patients with OSA remain undiagnosed and the majority of them are asymptomatic [3-8].

Although mounting evidence now suggest that OSA is independently associated with the development and progression of atherosclerosis, the prevalence of OSA in generalised systemic atherosclerosis has not been fully established [3, 9-12]. In order to further elucidate the link between OSA and clinical outcome in PAD patients, we decided to assess the prevalence and clinical correlates of previously unrecognised OSA in patients scheduled for lower limb surgical intervention for PAD. This study is part of a larger protocol in progress to evaluate the impact of sleep-time disorders on clinical outcome after vascular surgery in PAD patients.

\section{METHODS}

\section{Study population}

Patients aged $>40$ yrs with PAD referred to Turku University Hospital (Turku, Finland) for elective subinguinal revascularisation were eligible for this prospective cross-sectional study. Exclusion criteria were previously diagnosed OSA syndrome,
AFFILIATIONS

${ }^{*}$ Dept of Internal Medicine, Turku University Hospital,

${ }^{+}$Dept of Clinical Physiology, Turku University Hospital,

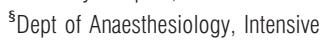
Care, Emergency Care and Pain

Medicine, Turku University Hospital,

**Dept of Surgery, Turku University Hospital,

${ }^{\#}$ Sleep Research Unit, University of Turku,

${ }^{f}$ Dept of Biostatistics, University of Turku, Turku, and

"Dept of Pulmonology, Tampere University Hospital, Tampere, Finland.

CORRESPONDENCE

T.T. Laitio

Dept of Anaesthesiology, Intensive

Care, Emergency Care and Pain

Medicine

Turku University Hospital

P.O.B. 52

FIN-20521 Turku

Finland

E-mail: timo.laitio@tyks.fi

Received:

Dec 292011

Accepted after revision:

May 272012

First published online:

June 142012 
congestive heart failure, atrial fibrillation or any other nonsinus rhythm, inability to co-operate, immobility, end-stage renal disease, history of coronary bypass within 3 yrs, or other major surgery within 3 months prior to enrolment. Between April 2006 and August 2010, 142 consecutive patients were considered eligible for the study and were contacted by a study nurse and inquired about their availability and interest in the study. 59 patients refused to give their consent mainly for logistic reasons and 84 patients gave their written informed consent and were enrolled. After rejection of two patients because of technically insufficient sleep studies, 82 patients were included in the final analyses. The study was approved by the Medical Ethics Committee of The Hospital District of Southwest Finland (based in Turku, Finland).

\section{Data collection}

All patients underwent a detailed clinical history and physical examination, including determination of body mass index, and measurement of waist circumference. Ankle-brachial index $(\mathrm{ABI})$ and toe pressures were used to assess lower limb circulation. Echocardiography was performed before polysomnography to determine left ventricular ejection fraction (LVEF). If ABI could not be measured (critical ischaemia) or if it indicated mediasclerosis ( $>1.3$ in diabetic patients), the values were omitted (table 1). Lipid profile was either determined preoperatively from fasting blood samples or collected from hospital records. A glucose tolerance test was performed in patients without a previous diagnosis of diabetes [13]. Coronary artery disease and hypertension were considered present if previously diagnosed with established criteria. Metabolic syndrome was diagnosed if at least three of the five following standard clinical criteria were present: elevated waist circumference (available in 76 patients) $(\geqslant 88 \mathrm{~cm}$ in females; $\geqslant 102 \mathrm{~cm}$ in males), elevated triglycerides $\left(\geqslant 1.7 \mathrm{mmol} \cdot \mathrm{L}^{-1}\right.$ or on drug treatment excluding statins), reduced high-density lipoprotein (HDL)-cholesterol $\left(<1.03 \mathrm{mmol} \cdot \mathrm{L}^{-1}\right.$ in males, $<1.3 \mathrm{mmol} \cdot \mathrm{L}^{-1}$ in females, or on drug treatment excluding statins), increased blood pressure ( $\geqslant 130 / 85$ or on drug treatment) and elevated fasting glucose $\left(\geqslant 5.6 \mathrm{mmol} \cdot \mathrm{L}^{-1}\right.$ or on drug treatment), as established by the American Heart Association and the National Heart, Lung and Blood Institute [14].

All subjects underwent an overnight polysomnography (Embla/Somnologica; MedCare, Reykjavik, Iceland) in a sleep laboratory [15]. Electroencephalogram (four channels), electrooculogram (two channels) and electromyogram (one channel) were recorded for determination of the sleep stages. Respiration was monitored with a pressure transducer attached to nasal prongs for respiratory flow. A pulse oximeter with finger probe (Nonin Medical Inc., Plymouth, MN, USA) measured arterial oxyhaemoglobin saturation. A separate fingertip oximeter was utilised to record plethysmographic signal for pulse transit time calculation. The Epworth sleepiness scale (ESS) and the Berlin Questionnaire (BQ) were used to assess symptoms of sleep apnoea $[16,17]$.

TABLE 1 Baseline characteristics according to severity of obstructive sleep apnoea (OSA)

\begin{tabular}{|c|c|c|c|c|c|}
\hline & All patients & No OSA & Mild OSA & Moderate OSA & Severe OSA \\
\hline Subjects n & 82 & 12 & 23 & 23 & 24 \\
\hline Age yrs & $67 \pm 9$ & $65 \pm 7$ & $64 \pm 10$ & $69 \pm 8$ & $70 \pm 8^{*}$ \\
\hline Male & $52(63)$ & $7(58)$ & $11(48)$ & $14(61)$ & $20(83)^{\bullet}$ \\
\hline Smoker & $31(38)$ & $7(58)$ & $11(48)$ & $5(22)$ & $8(33)$ \\
\hline Metabolic syndrome & $50(61)$ & $5(42)$ & $13(57)$ & $15(65)$ & $17(71)$ \\
\hline $\mathrm{BMI} \mathbf{k g} \cdot \mathrm{m}^{-2}$ & $27.0 \pm 4.1$ & $26.6 \pm 3.1$ & $26.9 \pm 4.5$ & $26.4 \pm 4.5$ & $27.8 \pm 3.7$ \\
\hline \multicolumn{6}{|l|}{ Waist circumference $\mathrm{cm}$} \\
\hline Male & $101 \pm 9$ & $102 \pm 6$ & $98 \pm 11$ & $102 \pm 12$ & $101 \pm 6$ \\
\hline Female & $102 \pm 12$ & $104 \pm 16$ & $105 \pm 9$ & $97 \pm 12$ & $98 \pm 15$ \\
\hline Diabetes mellitus & $35(43)$ & $6(50)$ & $11(48)$ & $11(48)$ & 7 (29) \\
\hline Hypertension & $62(76)$ & $8(67)$ & $14(61)$ & $20(87)$ & $20(83)$ \\
\hline CAD & $30(37)$ & $3(25)$ & $7(30)$ & $11(48)$ & $9(38)$ \\
\hline Stroke & $9(11)$ & $4(33)$ & $2(9)$ & $1(4)$ & $2(8)$ \\
\hline LVEF $^{\#} \%$ & $63(40-80)$ & $68(60-77)$ & $67(57-79)$ & $63(52-77)$ & $59(40-80)^{++}$ \\
\hline Cholesterol $\mathrm{mmol} \cdot \mathrm{L}^{-1}$ & $4.3 \pm 1.0$ & $4.3 \pm 1.2$ & $4.3 \pm 0.9$ & $4.5 \pm 1.0$ & $4.3 \pm 1.1$ \\
\hline $\mathrm{LDL}^{\bullet} \mathrm{mmol} \cdot \mathrm{L}^{-1}$ & $2.2 \pm 0.8$ & $2.1 \pm 0.9$ & $2.0 \pm 0.8$ & $2.3 \pm 0.5$ & $2.2 \pm 1.0$ \\
\hline $\mathrm{HDL} / \mathrm{Chol}^{+} \%$ & $34 \pm 10$ & $37 \pm 13$ & $37 \pm 9$ & $31 \pm 8$ & $32 \pm 10^{\circ}$ \\
\hline $\mathrm{TGL}^{\S} \mathrm{mmol} \cdot \mathrm{L}^{-1}$ & $1.7 \pm 1.0$ & $1.4 \pm 1.2$ & $1.6 \pm 1.0$ & $1.9 \pm 1.2$ & $1.7 \pm 1.0$ \\
\hline $\mathrm{ABI}$ ratiof & $0.57 \pm 0.21$ & $0.51 \pm 0.23$ & $0.63 \pm 0.18$ & $0.59 \pm 0.17$ & $0.56 \pm 0.25$ \\
\hline Toe pressure ${ }^{\# \#} \mathrm{mmHg}$ & $51 \pm 24$ & $44 \pm 25$ & $56 \pm 16$ & $49 \pm 17$ & $52 \pm 32$ \\
\hline PAD history yrs & $3.8 \pm 3.6$ & $3.1 \pm 3.4$ & $3.3 \pm 3.1$ & $4.3 \pm 4.1$ & $4.2 \pm 3.8$ \\
\hline Critical ischaemia & $10(12)$ & $4(33)$ & $0(0)$ & $2(9)$ & $4(17)$ \\
\hline
\end{tabular}

Data are presented as mean $\pm \mathrm{SD}$, mean (range) or $n(\%)$, unless otherwise stated. BMI: body mass index; CAD: coronary artery disease; LVEF: left ventricular ejection fraction; LDL: low density lipoprotein; HDL/Chol: high-density lipoprotein/total cholesterol ratio; TGL: triglycerides; ABI: ankle-brachial index; PAD: peripheral arterial disease. ${ }^{\#}: n=76 ;{ }^{\bullet}: n=75 ;{ }^{+}: n=80 ;{ }^{\S}: n=81 ;{ }^{f}: n=74 ;{ }^{\# \#}: n=77$. The significance of association with the severity of OSA was tested with univariate cumulative logistic regression analysis. $p$-values reflect the association of the variable with the severity of OSA. ${ }^{*}: p=0.02 ;{ }^{\bullet}: p=0.03 ;{ }^{++}: p=0.002$. 


\section{Data analysis}

The polysomnographic recordings, including sleep stages and arousals, were analysed by study physicians (K.T. Utriainen and $\mathrm{O}$. Polo) according to common guidelines. Apnoea was defined as an episode of complete cessation of airflow for $>10$ s. Hypopnoea was scored if a tidal volume reduction of $>50 \%$ for a minimum duration of $10 \mathrm{~s}$ was associated with a $4 \%$ decline in arterial oxyhaemoglobin saturation. The apnoea/ hypopnoea index (AHI) was defined as the number of the apnoea or hypopnoea episodes per hour of sleep. Arterial oxyhaemoglobin desaturation index was determined as the number of desaturations of $>4 \%$ per hour of sleep, and was used instead of the AHI in four patients in whom the nasal prongs had been detached during the recording. In these patients, the oximeter signal exhibited a desaturation-resaturation pattern typical for OSA. Sleep apnoea was considered significant when the AHI was $\geqslant 5$ episodes per hour. The severity of sleep apnoea was classified as mild (AHI 5-15), moderate (AHI 15-30) or severe (AHI >30) [15]. Central and obstructive episodes of apnoea were distinguished according to the respiratory swing observed in pulse transit time, as previously validated [18, 19]. Pulse transit time was calculated from the electrocardiogram and fingertip plethysmography signals using appropriate software (WinCPRS; Absolute Aliens, Turku, Finland).

\section{Statistical analysis}

The prevalence of OSA and each of its severity degree was determined and confidence intervals were calculated using binomial distribution. Significance of association of the severity of OSA (i.e. response variable) with each clinical variable shown in table 2 was first analysed using cumulative logistic regression models. The independent predictive value for the severity of OSA of each clinical variable was determined using a stepwise (variables with $\mathrm{p}>0.1$ significance of association excluded) ageand sex-adjusted multivariate analysis. Due to the small number of patients with no OSA, the significance of the predictors for the occurrence of OSA in general was not tested separately. Instead, the severity of OSA was used as an ordinal dependent variable that also included patients with no OSA. The cumulative odds ratio was calculated corresponding to a change equal to standard deviation of the predictor. The statistical analyses were performed with SAS 9.2 software for Windows (SAS Institute Inc., Cary, NC, USA).

\section{RESULTS}

Demographic features and clinical data of the patients are summarised in table 1 . Sleep variables are described in table 2 and details of long-term medication in table S3. As expected, most of the patients had other manifestations of cardiovascular disease or their risk factors. Although most of the patients were nonobese, metabolic syndrome was common but was not related to of the severity of OSA. All of the included subjects were outpatients and most of them were independent in everyday activities. Eligible patients refusing their consent were significantly older (aged 71 yrs versus 67 yrs; $p=0.02$ ), but did not differ in any of the available clinical parameters (data not shown).

Sleep apnoea (AHI $\geqslant 5$ events per hour of sleep) was observed in 70 (85\%; $95 \%$ CI $76-92 \%)$ out of 82 patients. OSA was mild in $23(28 \%)$, moderate in $23(28 \%)$ and severe in $24(29 \%)$ subjects. Oxyhaemoglobin desaturation index (mean $23 \mathrm{~h}^{-1}$, interquartile range 8-35) and the $\mathrm{AHI}$ (mean $24 \mathrm{~h}^{-1}$, interquartile range 8-36) had a high correlation in the whole study population (Spearman's coefficient $r=0.93$ ). The episodes of apnoea were predominantly obstructive (proportion of central apnoea 0 $40 \%$ and mean $23 \%$, in all patients from all respiratory events). The proportion of central apnoea was similar in all categories of OSA. LVEF was mildly decreased (40-49\%) in four patients with severe OSA; the rest of the patients had normal LVEF $(>50 \%)$.

The ESS and BQ scores were low in the majority of the patients and failed to correlate with the severity of OSA (table 2). The ESS was pathological $(\geqslant 11)$ in five patients with mild OSA, two with moderate OSA and one with no OSA.

The LVEF and HDL/total cholesterol ratio (HDL/Chol), lowest arterial oxygen saturation $\left(\mathrm{Sa}_{2} \mathrm{O}_{2} \mathrm{Nadir}\right)$, male sex and age were the only variables associated with the severity of OSA (table 1), but no relation to obesity, metabolic syndrome or any manifestation of atherosclerosis, other than PAD, was observed. In this patient group limited to severe PAD there

TABLE 2 Sleep variables according to severity of obstructive sleep apnoea (OSA)

\begin{tabular}{|c|c|c|c|c|c|}
\hline & All patients & No OSA & Mild OSA & Moderate OSA & Severe OSA \\
\hline Subjects $\mathbf{n}$ & 82 & 12 & 23 & 23 & 24 \\
\hline BQ 2-3 $3^{\#}$ & $36(44)$ & $4(33)$ & 9 (39) & $12(52)$ & $11(46)$ \\
\hline ESS score ${ }^{\#}$ & $5.2(0-17)$ & $4.8(1-12)$ & $5.9(0-17)$ & $5.7(0-11)$ & $4.2(0-10)$ \\
\hline EDS (ESS > 10) & $8(10)$ & $1(8)$ & $5(22)$ & $2(9)$ & $0(0)$ \\
\hline Arousal index events $\cdot h^{-1}$ & $21 \pm 10$ & $13 \pm 7$ & $17 \pm 9$ & $22 \pm 10$ & $27 \pm 9^{\circ}$ \\
\hline $\mathrm{Sa}_{1} \mathrm{O}_{2} \mathrm{~T} 90 \mathrm{~min}$ & $29 \pm 59$ & $14 \pm 32$ & $20 \pm 46$ & $15 \pm 32$ & $57 \pm 85^{\S}$ \\
\hline
\end{tabular}

Data are presented as $n(\%)$, mean (range) or mean \pm SD, unless otherwise stated. BQ 2-3: Berlin Questionnaire ( $\geqslant 2$ positive categories indicate a high risk of OSA); ESS: Epworth sleepiness scale; EDS: excessive daytime sleepiness; $\mathrm{Sa}, \mathrm{O}_{2}$ : arterial oxygen saturation; $\mathrm{Sa}, \mathrm{O}_{2}$ T90: cumulative time for $<90 \% \mathrm{Sa}, \mathrm{O}_{2}$. The significance of association with the severity of OSA was tested with univariate cumulative logistic regression analysis. $\mathrm{p}$-values reflect the association of the variable with the severity of OSA. ${ }^{\#}: n=79 ;{ }^{+}: p=0.0001 ;{ }^{\bullet}: p<0.0001 ;{ }^{\S}: p=0.02$. 
was no correlation between the AHI and the severity of PAD, as measured by $\mathrm{ABI}$ and toe pressures. In the age- and sexadjusted multivariate analysis (arousal index, $\mathrm{Sa}_{\mathrm{a}} \mathrm{O}_{2}$ nadir and time below 90\% saturation excluded), LVEF (cumulative OR $0.44,95 \%$ CI $0.26-0.72 ; \mathrm{p}=0.002$, corresponding to a change equal to SD $8 \%$-point) and HDL/Chol (cumulative OR 0.58, $95 \%$ CI $0.37-0.90 ; p=0.03$, corresponding to SD $10 \%$-point) remained significant independent predictors for the severity of OSA. Decreasing percentages in both parameters marked the worsening of OSA.

\section{DISCUSSION}

The main finding of this study was that the prevalence of sleep apnoea is unexpectedly high in patients with PAD requiring surgical intervention. Diagnosed sleep apnoea was mainly obstructive and unrelated to the clinical signs (either obesity or metabolic syndrome), or to the symptoms of the conventional OSA syndrome (excessive sleepiness).

Sleep apnoea in cardiovascular disease is conventionally linked with congestive heart failure. Following our exclusion criteria, none of the patients had clinical heart failure and only four patients had mild left ventricular systolic dysfunction. Nevertheless, decreasing LVEF remained as an independent predictor of the AHI even in this carefully selected population. This finding may support the earlier observations showing that OSA impairs left ventricular function [20]. It is also notable that decreasing LVEF was associated with the worsening of OSA despite remaining mostly in the normal range.

Obesity is considered the single major risk factor for OSA. It has been suggested that OSA is a manifestation of the metabolic syndrome and potentiates its effect on the overall atherosclerotic burden [21, 22]. However, most patients in our study were not obese. This is in line with earlier observations on clinical characteristics of PAD [23]. Although more than half of our patients had metabolic syndrome, neither its prevalence nor the patients' waist circumference correlated with the severity of OSA suggesting that OSA is not a manifestation of metabolic syndrome in this patient population. In addition, current multivariate analysis did not show any relationship between the severity of OSA and any manifestation of atherosclerosis (e.g. hypertension, coronary artery disease or stroke) other than PAD. Accordingly, none of the comorbidities or cardiovascular risk factors in this study explains the high prevalence of OSA. Consequently, the aetiology of OSA in this patient group remains undetermined.

Mounting evidence show that sleep apnoea enhances endothelial dysfunction and arterial stiffness, the key factors in the development of atherosclerosis [10-12, 24-26]. The prevalence of sleep apnoea in our PAD patients is higher than in any other previously reported population with manifestations of atherosclerosis, with earlier studies showing $30-58 \%$ prevalence of OSA in coronary artery disease and $30-80 \%$ prevalence in arterial hypertension [3, 4, 8]. Current results support these earlier studies, showing a close relationship between atherosclerosis and OSA. However, reasons for the observed high prevalence can only be speculated as our study design does not allow any conclusions on causal relationships. According to a recent study, serious incident cardiovascular disease can exacerbate sleep apnoea implying a reversed causal pathway [27].
An alternate aetiology to simple mechanical airway obstruction is also supported by this study, revealing a higher proportion of central apnoea than has been found in earlier studies [28]. It has been shown that cerebral white matter lesions are associated with the common risk factors and manifestations of atherosclerosis, including compromised lower limb circulation [29]. Furthermore, white matter disease is known to be related to central sleep apnoea [30]. Therefore, the presence of asymptomatic ischaemic brain lesions may account for the increased proportion of central apnoea episodes, but this does not explain the high prevalence of sleep-disordered breathing per se. Further studies are warranted to establish the aetiology of OSA in patients with severe atherosclerosis.

There are important limitations to be considered. The study design was cross-sectional without any possibility to establish causality. Furthermore, it is difficult to assess the association of OSA and PAD independent of age and the co-morbidities that are commonly associated with both conditions. Due to strict exclusion criteria, our study sample is small and represents a subgroup of patients with PAD with a limited degree of comorbidity. Therefore, our results cannot be directly extrapolated to the general clinical population of PAD patients. The modest size of the study also limits the weight of statistical conclusions. Our strict exclusion policy aimed at a homogenous population without confounding factors, excluding the oldest patients and the most severe cases of PAD, as well as all patients with clinical heart failure who are likely to have a significant prevalence of central sleep apnoea [3]. Taken together, the potential biases arising from patient selection should underestimate instead of overestimating the real clinical prevalence of sleep apnoea in PAD patients. However, systemic atherosclerosis was determined solely by the presence of PAD; the status of other vascular beds (e.g. coronary, carotid and renal) was not specifically evaluated. The exact nature of apnoea in this population must also be interpreted with caution due to the considerable proportion of central episodes.

We conclude that asymptomatic OSA is exceedingly common and often under-recognised in severe PAD. Studies in larger populations and outcome data are warranted to clarify the significance of this finding.

\section{SUPPORT STATEMENT}

This study has been funded by GE Healthcare Finland, Finnish Funding Agency for Technology and Innovation (TEKES), Clinical Research Fund (EVO) of Turku University Hospital and Instrumentarium Foundation.

\section{CLINICAL TRIAL}

This study is registered at www.ClinicalTrials.gov with identifier number NCT00712946.

\section{STATEMENT OF INTEREST}

A statement of interest for T.M. Salo and the study itself can be found at www.erj.ersjournals.com/site/misc/statements.xhtml

\section{ACKNOWLEDGEMENTS}

We thank N. Karppinen (University of Turku, Sleep Research Unit, Turku, Finland) and K. Leivo (Dept of Anaesthesiology, Intensive Care, Emergency Care and Pain Medicine, Turku University Hospital, 
Turku) for technical support. We further thank M. Salmenperä (Dept of Anaesthesiology and Intensive Care, Helsinki University Hospital, Helsinki, Finland) and M. Lepäntalo (Dept of Vascular Surgery, Helsinki University Hospital) for critical comments to the manuscript.

\section{REFERENCES}

1 Hirsch AT, Criqui MH, Treat-Jacobson D, et al. Peripheral arterial disease detection, awareness and treatment in primary care. JAMA 2001; 286: 1317-1324.

2 Conte MS, Belkin M, Upchurch GR, et al. Impact of increasing comorbidity on infrainguinal reconstruction: a 20-year perspective. Ann Surg 2001; 233: 445-452.

3 Bradley TD, Floras JS. Obstructivesleep apnoea and its cardiovascular consequences. Lancet 2009; 373: 82-93.

4 Duran J, Esnaola S, Rubio R, et al. Obstructive sleep apnoeahypopnoea and related clinical features in a population-based sample of subjects aged 30 to 70 yr. Am J Respir Crit Care Med 2001; 163: 685-689.

5 Yaggi HK, Concato J, Kernan WN, et al. Obstructive sleep apnea as a risk factor for stroke and death. $N$ Engl J Med 2005; 353: 2034-2041.

6 Marin JM, Carrizo SJ, Vicente E, et al. Long-term cardiovascular outcomes in men with obstructive sleep apnoea-hypopnoea with or without treatment with continuous positive airway pressure: an observational study. Lancet 2005; 365: 1046-1053.

7 Young T, Evans L, Finn L, et al. Estimation of the clinically diagnosed proportion of sleep apnea syndrome in middle-aged men and women. Sleep 1997; 20: 705-706.

8 Peppard PE, Young T, Palta M, et al. Prospective study of the association between sleep-disordered breathing and hypertension. N Engl J Med 2000; 342: 1378-1384.

9 Drager LF, Bortolotto LA, Krieger EM, et al. Additive effects of obstructive sleep apnea and hypertension on early markers of carotid atherosclerosis. Hypertension 2009; 53: 64-69.

10 Jelic S, Padeletti M, Kawut SM, et al. Inflammation., oxidative stress and repair capacity of the vascular endothelium in obstructive sleep apnea. Circulation 2008; 117: 2270-2278.

11 Mason RH, Ruegg G, Perkins J, et al. Obstructive sleep apnea in patients with abdominal aneurysms: highly prevalent and associated with aneurysm expansion. Am J Respir Crit Care Med 2011; 183: 668-674.

12 Drager LF, Bortolotto LA, Figueiredo AC, et al. Effects of continuous positive airway pressure on early signs of atherosclerosis in obstructive sleep apnea. Am J Respir Crit Care Med 2007; 176: 706-712.

13 American Diabetes Association. Diagnosis and classification of diabetes mellitus. Diabetes Care 2008; 31: Suppl. 1, S55-S60.

14 Grundy SM, Cleeman JI, Daniels SR, et al. Diagnosis and management of the metabolic syndrome: an American Heart Association/National Heart, Lung and Blood Institute scientific statement. Circulation 2005; 112: 2735-2752.
15 American Academy of Sleep Medicine Task Force. Sleep-related breathing disorders in adults: recommendations for syndrome definition and measurement techniques in clinical research. The report of an American Academy of Sleep Medicine Task Force. Sleep 1999; 22: 667-689.

16 Johns MW. A new method for measuring daytime sleepiness: the Epworth sleepiness scale. Sleep 1991; 14: 540-545.

17 Netzer NC, Stoohs RA, Netzer CM, et al. Using the Berlin Questionnaire to identify patients at risk for the sleep apnea syndrome. Ann Intern Med 1999; 131: 485-491.

18 Argod J, Pépin JL, Lévy P. Differentiating obstructive and central sleep respiratory events through pulse transit time. Am J Respir Crit Care Med 1998; 158: 1778-1783.

19 Argod J, Pépin JL, Smith RP, et al. Comparison of esophageal pressure with pulse transit time as a measure of respiratory effort for scoring nonapneic respiratory events. Am J Respir Crit Care Med 2000; 162: 87-93.

20 Laaban JP, Pascal-Sebaoun S, Bloch E, et al. Left ventricular dysfunction in patients with obstructive sleep apnea syndrome. Chest 2002; 122: 1133-1138.

21 Vgontzas AN, Bixler EO, Chrousos GP. Sleep apnea is a manifestation of the metabolic syndrome. Sleep Med Rev 2005; 9: 211-224.

22 Drager LF, Bortolotto LA, Maki-Nunes C, et al. The incremental role of obstructive sleep apnoea on markers of atherosclerosis in patients with metabolic syndrome. Atherosclerosis 2010; 208: 490-495.

23 Meijer WT, Hoes AW, Rutgers D, et al. Peripheral arterial disease in the elderly. The Rotterdam Study. Arterioscler Thromb Vasc Biol 1998; 18: 185-192.

24 Ip MS, Tse HF, Lam B, Tsang KW, et al. Endothelial function in obstructive sleep apnea and response to treatment. Am J Respir Critic Care Med 2004; 169: 348-353.

25 Kohler M, Craig S, Nicoll D, et al. Endothelial function and arterial stiffness in minimally symptomatic sleep apnea. Am J Respir Crit Care Med 2008; 178: 984-988.

26 Herrington DM, Brown WV, Mosca L, et al. Relation between arterial stiffness and subclinical aortic atherosclerosis. Circulation 2004; 110: 432-437.

27 Chami HA, Resnick HE, Quan SF, et al. Association of incident cardiovascular disease with progression of sleep-disordered breathing. Circulation 2011; 123: 1280-1286.

28 Ferrier K, Campbell A, Yee B, et al. Sleep-disordered breathing occurs frequently in stable outpatients with congestive heart failure. Chest 2005; 128: 2116-2122.

29 Longstreth WT Jr, Arnold AM, Beauchamp NJ Jr, et al. Incidence, manifestations and predictors of worsening white matter on serial cranial magnetic resonance imaging in the elderly: the cardiovascular health study. Stroke 2005; 36: 56-61.

30 Robbins J, Redline S, Ervin A, et al. Associations of sleepdisordered breathing and cerebral changes on MRI. J Clin Sleep Med 2005; 1: 159-165. 